\title{
Is TIMP-1 immunoreactivity alone or in combination with other markers a predictor of benefit from anthracyclines in the BR9601 adjuvant breast cancer chemotherapy trial?
}

Alison F Munro ${ }^{6}$, Annette Bartels², Eva Balslev ${ }^{3}$, Christopher J Twelves ${ }^{4}$, David A Cameron ${ }^{5}$, Nils Brünner ${ }^{2}$ and John MS Bartlett ${ }^{1,6^{*}}$

\begin{abstract}
Introduction: Predictive cancer biomarkers to guide the right treatment to the right patient at the right time are strongly needed. The purpose of the present study was to validate prior results that tissue inhibitor of metalloproteinase 1 (TIMP-1) alone or in combination with either HER2 or TOP2A copy number can be used to predict benefit from epirubicin (E) containing chemotherapy compared with cyclophosphamide, methotrexate and fluorouracil (CMF) treatment.

Methods: For the purpose of this study, formalin fixed paraffin embedded tumor tissue from women recruited into the BR9601 clinical trial, which randomized patients to E-CMF versus CMF, were analyzed for TIMP-1 immunoreactivity. Using previously collected data for HER2 amplification and TOP2A gene aberrations, we defined patients as "anthracycline non-responsive", that is, 2T (TIMP-1 immunoreactive and TOP2A normal) and HT (TIMP-1 immunoreactive and HER2 negative) and anthracycline responsive (all other cases).

Results: In total, 288 tumors were available for TIMP-1 analysis with (183/274) 66.8\%, and (181/274) 66.0\% being classed as $2 \mathrm{~T}$ and HT responsive, respectively. TIMP-1 was neither associated with patient prognosis (relapse free survival or overall survival) nor with a differential effect of E-CMF and CMF. Also, TIMP-1 did not add to the predictive value of HER2, TOP2A gene aberrations, or to Ki67 immunoreactivity.
\end{abstract}

Conclusion: This study could not confirm the predictive value of TIMP-1 immunoreactivity in patients randomized to receive E-CMF versus CMF as adjuvant treatment for primary breast cancer.

\section{Introduction}

A number of clinical studies have clearly indicated the superiority of anthracycline-containing chemotherapy over the combination of cyclophosphamide, methotrexate and 5-flourouracil (CMF) in adjuvant treatment of breast cancer [1-3]. However, a significant number of anthracycline treated patients will experience disease recurrence, suggesting that their breast cancers contained tumor cells refractory to adjuvant anthracyclines. Moreover, patients receiving an anthracycline may

\footnotetext{
* Correspondence: John.Bartlett@oicr.on.ca

'Transformative Pathology, Ontario Institute for Cancer Research, MaRS Centre, South Tower, 101 College Street, Suite 800, Toronto, ON M5G 0A3, Canada

Full list of author information is available at the end of the article
}

experience significant toxicity during treatment [4] With a validated predictive biomarker for anthracycline sensitivity/resistance, it would be possible to direct the toxic adjuvant anthracycline treatment to those patients with the highest likelihood of a treatment benefit while those patients with anthracycline resistant tumors could receive an alternative treatment, for example, a taxane.

A number of studies have suggested that breast cancer patients with HER2 positive tumors, those amplified and/ or overexpressing HER2, are those obtaining the greatest benefit from the addition of an anthracycline [5]. Similar data have been presented for the Topoisomerase II $\alpha$ (TII $\alpha)$ gene copy number (TOP2A) or enzyme, the latter being a target of the anthracyclines [6]. More recently, 
we have shown that tumor levels of other members of the HER family may be associated with benefit from adjuvant chemotherapy [7]. However, these effects are not substantiated in a recent meta-analysis of multiple trials with data available for HER2 and TOP2A [8]. Emerging data may suggest that novel markers associated with centromeric enumeration probe for chromosome 17 (CEP17) duplication may identify, in part, those patients with anthracycline responsive cancer [9]. However, increasingly, there is recognition of the complex nature of tumor resistance to chemotherapy and the need for multiple markers to stratify patients according to their likelihood of response to chemotherapy.

Tissue inhibitor of metalloproteinase 1 (TIMP-1) protein as determined by immunhistochemistry is another potential molecular marker for anthracycline benefit [10]. Preclinical data linked TIMP-1 to protection against chemotherapy-induced inhibition of apoptosis [11,12], and when applying TIMP-1 immunohistochemistry (IHC) to tissue microarrays (TMAs) from the Danish Breast Cancer Cooperative Group (DBCG) 89D prospective randomized adjuvant trial (cyclophosphamide, epirubicin, 5-fluorouracil (CEF) vs CMF), [10], it was demonstrated that women with breast tumors displaying cancer cell TIMP-1 immunoreactivity had similar benefit from adjuvant chemotherapy regardless of the addition of an anthracycline, while women lacking TIMP-1 immunoreactivity in the cancer cells had a significant improved benefit (increased disease free survival (DFS) and overall survival (OS)) when receiving combination therapy with an anthracycline as compared with women who received treatment with CMF [10]. A subsequent study, including the same DBCG $89 \mathrm{D}$ patient cohorts, showed that TIMP-1 immunhistochemistry could be combined with the HER2 or TOP2A gene copy number forming a biomarker panel which could predict anthracycline benefit in almost double the number of patients as each of these markers could do separately [13]. In a more recent study [14], including patient samples (TMAs) from the Canadian MA5 study in which patients were randomized to receive either CEF or CMF [14], we reported on a substantial reduction in mortality by CEF compared to CMF in patients with a HER2/TIMP-1 or TOP2A/TIMP-1 responsive profile; however, we could not show a similarly significant reduction in recurrence-free survival events, where a benefit of CEF over CMF was found irrespective of TIMP-1 status.

To further test the hypothesis that TIMP-1 in combination with either HER2 or TOP $2 A$ copy number can be used to predict benefit from adjuvant chemotherapy, including an anthracycline, we assessed TIMP-1 immunoreactivity in TMAs obtained from the BR9601 study in which patients were randomized to receive either epirubicin followed by CMF (E-CMF) or CMF alone [2]. On all samples, data on tumor genetic alterations of HER 2 and TOP2A and protein expression of HER2 and Ki67 were available [7].

\section{Materials and methods \\ Patients}

The BR9601 study recruited 374 pre- and post-menopausal women with completely excised, histologically confirmed early breast cancer that had a clear indication for adjuvant chemotherapy according to current UK practice, which relies on the Nottingham Prognostic Index for risk stratification. For further details, please see [2]. The patients were randomized between the standard arm of eight cycles of CMF (intravenous (i.v.) cyclophosphamide $750 \mathrm{mg} / \mathrm{m}^{2}$, methotrexate $50 \mathrm{mg} / \mathrm{m}^{2}$ and 5 -fluorouracil $600 \mathrm{mg} / \mathrm{m}^{2}$ ) every 21 days, and E-CMF (four cycles of $100 \mathrm{mg} / \mathrm{m}^{2}$ of epirubicin every 21 days followed by four cycles of the same CMF regimen). The protocol was approved by central and local ethics committees, and each patient provided written informed consent prior to randomization. The primary outcomes of the BR9601 study were relapse free (RFS) and OS (2). Patients were followed for a mean of 5.30 years (range 2.76 to 8.51 years).

For the current analysis, following approval by the central ethics committee (South East Multi-centre Research Ethics Committee), tissue blocks were retrieved from 321 cases (85.8\%) among which 288 cases $(77.0 \%)$ were applicable for the present study. A total of 30 samples were lost on the TMAs and 3 cases had incomplete follow-up. Triplicate tissue microarrays (TMAs) with $0.6 \mathrm{~mm}^{2}$ cores were constructed using cores taken from the middle of the invasive tumor following review by a pathologist (JST). Duplicate TMAs were used for the purpose of this study.

\section{Triple color fluorescent in situ hybridization (FISH)}

FISH was performed using a triple-color probe for HER2, Topoisomerase II $\alpha$ (TOP2A) and chromosome 17 (CEP17) (Abbot Molecular, Maidenhead, Berkshire, UK) as previously described [15-17]. Amplifications were defined as gene:chromosome ratios $>1.5$ for TOP $2 A$ and $>2.0$ for HER2. TOP $2 A$ deletions were defined as gene:chromosome ratios $<0.8$ [17].

\section{Immunohistochemistry}

The TIMP-1 immunohistochemistry procedure used has previously been described in details [18]. In brief, TMAs were deparaffinized in xylene and rehydrated in graded concentrations of ethanol. For antigen retrieval, the sections were microwave treated in citrate buffer pH6 and endogenous peroxidase activity was blocked by hydrogen peroxide. IHC staining for TIMP-1 was performed 
overnight at $4{ }^{\circ} \mathrm{C}$ and used the mouse monoclonal antibody, clone VT7 [19] raised against recombinant human TIMP-1 $(0.25 \mu \mathrm{g} / \mathrm{ml})$.

TIMP-1 staining was scored by two experienced observers ( $\mathrm{AB}$ and $\mathrm{EB})$ blinded to patient identity and outcome. If any TIMP-1 positive cells were evident in the section it was scored as positive, thus following the same procedure as in our previous studies $[10,13]$.

The IHC procedure for Ki67 (clone MIB-1, Dako, Glostrup, Denmark) has been described previously [20]. Staining was scored by a single experienced scorer (AM) blinded to patient identity and outcome and counting the percentage of Ki67 positive cells. For Ki67 13\% positivity was used as a cut point for dividing samples into Ki67 low and high, respectively [20], consistent with our previous studies.

\section{Statistics}

The IBM SPSS (v14) statistical software (IBM corporation, Portsmouth, Hampshire, UK) was used for statistical analysis. Kaplan-Meier estimates of survival were used for analysis of RFS and OS. The Cox's proportional hazard model was used to obtain hazard ratios for relapse or death. When comparing outcomes between the treatment arms within the two groups of patients identified by biomarker expression, formal $P$-values were not calculated as in most cases, one group was much smaller than the other. The Cox model was instead used to identify statistically significant interactions between biomarkers (expression or gene alterations) and outcome on the different treatments (treatment by marker effect), in models that also included biomarker status (marker effect) and treatment, as covariates.

RFS was calculated from the date of randomization to the date of relapse or the date last seen. OS was calculated from the date of randomization to the date of breast cancer specific death or the date last seen.

\section{Results}

There were no significant differences in patient baseline characteristics between the overall BR9601 trial population $(n=374)$ and the population $(n=288)$ included in this biomarker study (Table 1). Of 288 patients included in the current study, 96 (33.3\%) relapsed and 78 (27.1\%) died during the follow-up period. A survival analysis of these 288 patients confirmed the advantage of E-CMF over CMF observed in the main trial [2] with HR: $0.57 ; 95 \%$ CI 0.37 to $0.86 ; P=0.006$ and HR: $0.64 ; 95 \%$ CI 0.41 to 1.01 ; $P=0.05$ for RFS and OS, respectively. All subsequent analyses are restricted to these 288 cases, or sub-sets thereof.

\section{TIMP1 immunoreactivity}

Successful TIMP-1 staining was achieved for 291/321 cases. Three cases were lost to follow-up and excluded
Table 1 Patient/tumor characteristics from the BR9601 trial and samples retrieved for the current study

\begin{tabular}{lll}
\hline & BR9601 & TIMP-1 Analysis \\
\hline Number & 374 & 288 \\
Age & $50.9(44.7$ to 56.6$)$ & $50.5(45.0$ to 57.0$)$ \\
E-CMF (1) & $183(48.9 \%)$ & $140(48.6 \%)$ \\
CMF (2) & $191(51.1 \%)$ & $148(51.4 \%)$ \\
Tumor size (median) & $23(17$ to 30$)$ & $25(17$ to 30$)$ \\
Positive nodes & $2(1$ to 4$)$ & $3(1$ to 4$)$ \\
Grade & $3(2$ to 3$)$ & $3(2$ to 3$)$ \\
NPI & $5.30(4.50$ to 5.61$)$ & $5.21(4.50$ to 5.60$)$ \\
ER +ve & $202(54.0 \%)$ & $158(54.9 \%)$ \\
ER -ve & $119(31.8 \%)$ & $96(33.3 \%)$ \\
ER Unk & $53(14.2 \%)$ & $34(11.8 \%)$ \\
\hline
\end{tabular}

from subsequent analysis. Some TIMP-1 positive tumors displayed a homogenous staining, while others presented with a heterogenous staining pattern (Figure 1). Of the 288 cases available for analysis: $42 \%$ of the tumors had one or two TIMP-1 positive cores while $58 \%$ presented with two TIMP-1 negative cores.

\section{HER2, TOP2A and Ki67}

Results for HER2 and TOP2A gene copy analyses and Ki67 protein analysis have been previously presented and are summarized in Table 2.

\section{Associations between TIMP-1 and Ki67 immunoreactivity and HER2 and TOP2A gene copy numbers and RFS and OS} The prognostic significance of each of the biological markers included in this study was first tested on the entire included patient cohort $(n=288)$, irrespective of their allocated adjuvant chemotherapy. Exploratory analysis of these markers (minus TIMP-1) showed the results to be similar in this cohort and the 321 patients included in the original biomarker study [7]. TIMP-1 immunoreactivity was not significantly associated with RFS (HR $=0.83 ; 95 \%$ CI: 0.55 to $1.24 ; P=0.36$ ) or OS (HR $=0.69 ; 95 \%$ CI: 0.43 to $1.09 ; P=0.11)$. However, TIMP-1 positivity was significantly associated with an increased number of positive lymph nodes $(P=0.01)$, ER $\alpha$ positive disease $(P=0.004)$, and decreased proliferation (measured by Ki67; $P=0.004$; Table 3).

Tumors were classified as $2 \mathrm{~T}$ responsive (TOP2A abnormal and/or TIMP-1 negative) and $2 \mathrm{~T}$ non-responsive (TOP2A normal and TIMP-1 immunoreactive) [13]. Using this definition, 183/274 (66.8\%) of tumors were classed as $2 \mathrm{~T}$ responsive and $91 / 274(33.2 \%)$ as $2 \mathrm{~T}$ nonresponsive. There was no significant difference in RFS $(\mathrm{HR}=0.91 ; 95 \% \mathrm{CI}: 0.585$ to $1.417 ; P=0.68)$ and $\mathrm{OS}$ $(\mathrm{HR}=0.773 ; 95 \% \mathrm{CI}: 0.470$ to $1.271 ; P=0.30)$ between patients with the $2 \mathrm{~T}$ responsive profile when compared to those with the non-responsive profile. A $2 \mathrm{~T}$ responsive 


\section{Panel A: Homogenous staining,}

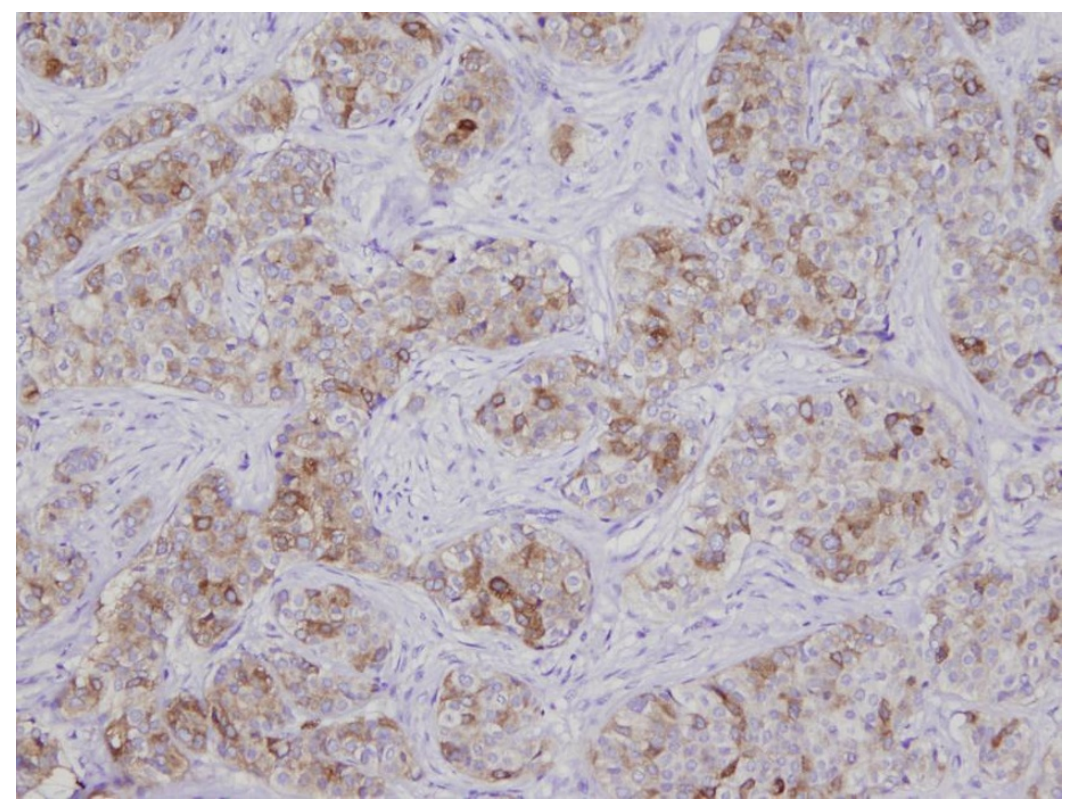

\section{Panel B: Heterogeneous staining.}

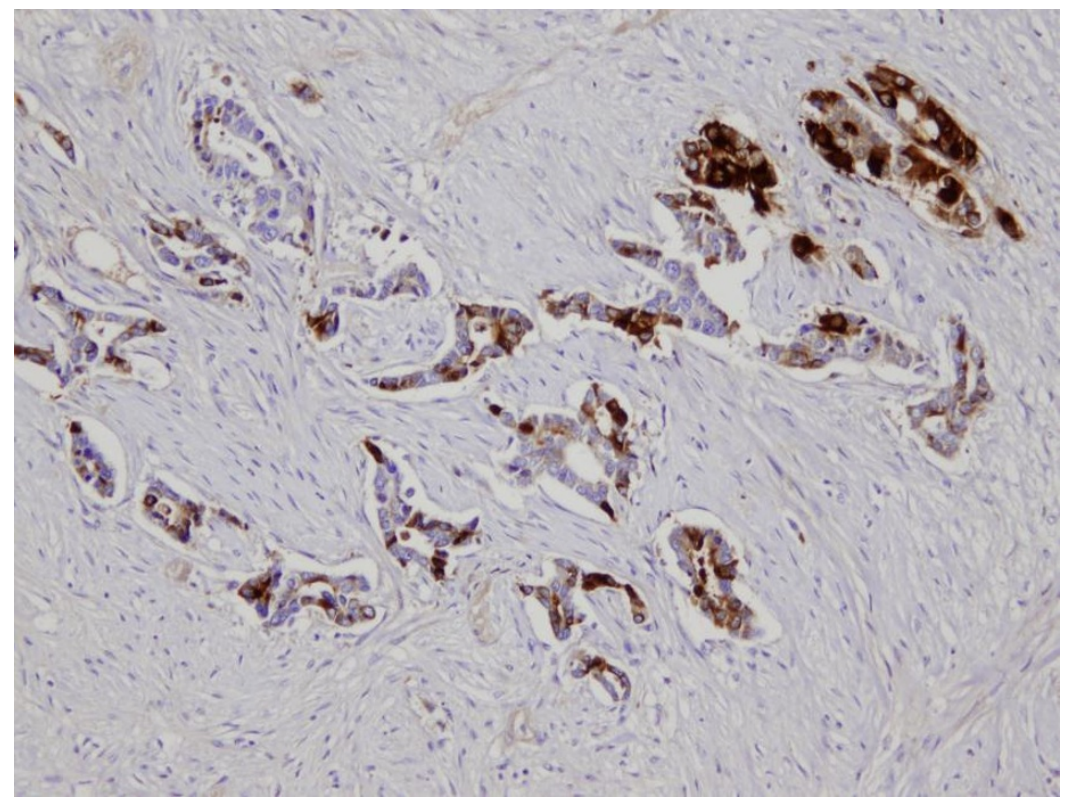

Figure 1 Illustrative figures of TIMP1 staining. Panel A: Homogenous staining. Panel B: Heterogeneous staining.

profile was associated with ER $\alpha$ negative disease $(P=$ $0.0001)$, increased pathological grade $(P=0.005)$, and increased proliferation $(P=0.001)$.

On the basis of TIMP-1 immunoreactivity and HER2 gene copy numbers, 181 (66\%) of tumors were classified as HT responsive (HER2 amplified and/or TIMP-1 negative) and 93 (34\%) as HT non-responsive (HER2 negative and TIMP-1 immunoreactive). Patients who had an HT responsive profile had a significantly decreased RFS (HR $=1.64 ; 95 \% \mathrm{CI}: 1.035$ to $2.6 ; P=$ 0.037) and OS (HR: 2.08; 95\% CI: 1.21 to 3.56 ; $P=$ 0.007 ) when compared to those with a non-responsive 
Table 2 HER2 and TOP2A gene status and Ki67 protein expression in the BR9601 cohort used for this study

\begin{tabular}{|c|c|c|c|c|}
\hline & & Amplified & Deleted & Normal \\
\hline HER2 FISH & $274 / 288(95.1 \%)$ & $62(22.6 \%)$ & & $212(77.4 \%)$ \\
\hline \multirow[t]{2}{*}{ TOP2A FISH } & $274 / 288(95.1 \%)$ & 25 (9.1\%) & 48 (17.5\%) & 201 (73.4\%) \\
\hline & & High & Low & \\
\hline Ki67 & 284/288 (98.6\%) & $120(42.3 \%)$ & $164(57.7 \%)$ & \\
\hline
\end{tabular}

Table 3 Associations between TIMP-1, Ki-67, HER2 and TOP2A

\begin{tabular}{|c|c|c|c|c|}
\hline & & TIMP-1 Negative & TIMP-1 Positive & $P$-value \\
\hline \multirow[t]{2}{*}{ Ki67 } & Low & $83(50.6 \%)$ & $81(67.5 \%)$ & 0.004 \\
\hline & High & 81 (49.4\%) & $39(32.5 \%)$ & \\
\hline \multirow[t]{2}{*}{ HER2 } & Normal* & 119 (75.8\%) & $93(79.5 \%)$ & 0.470 \\
\hline & Amplified & $38(24.2 \%)$ & $24(20.5 \%)$ & \\
\hline \multirow[t]{3}{*}{ TOP2A } & Deleted & $32(20.4 \%)$ & $16(13.7 \%)$ & 0.310 \\
\hline & Normal & 110 (70.1\%) & $91(77.8 \%)$ & \\
\hline & Amplified & 15 (9.6\%) & $10(8.5 \%)$ & \\
\hline
\end{tabular}

Normal includes samples with HER2 deletions and HER2 normal samples.

profile. A HT responsive profile was associated with ER $\alpha$ negative disease $(P=0.0002)$, increased pathological grade $(P=0.003)$, and increased proliferation $(P=$ 0.0002).

Treatment by marker analysis of the influence of TIMP-1, TOP2A and HER2 on RFS and OS benefits of E-CMF over CMF Subsequent analyses focused on possible differential effects of the expression of these markers on RFS and OS between patients receiving E-CMF and those treated with CMF alone. The results for HER2 and TOP2A gene copy number counts have been published previously [7].

Hazard ratios for TIMP-1 immunoreactivity and associated profiles between patients receiving E-CMF and CMF alone are summarized in Table 4 . Treatment by marker (TxM) HRs demonstrates that there is no evidence that lack of TIMP-1 immunoreactivity alone or in combination with TOP2A or HER2 gene aberrations is predictive of anthracycline benefit. In patients with TIMP-1 immunoreactivity there is a trend towards an increase in RFS $(\mathrm{HR}=0.48$; 95\% CI: 0.24 to 0.93; Figure $2 \mathrm{~A})$ and OS (HR $=0.46$; $95 \%$ CI: 0.21 to 1.00$)$ in patients treated with ECMF compared to CMF alone. However, a similar trend for RFS (HR $=0.64 ; 95 \% \mathrm{CI}$ : 0.38 to 1.10 ; Figure $2 \mathrm{~B})$ and
OS ( $\mathrm{HR}=0.78 ; 95 \% \mathrm{CI}: 0.45$ to 1.38$)$ was apparent in patients whose tumors were negative for TIMP-1 suggesting the trend is associated with benefit from treatment irrespective of the immunoreactivity of TIMP-1.

\section{Discussion}

This study, which included 288/374 of the patients enrolled in the original BR9601 adjuvant study [2], failed to confirm the predictive value of TIMP-1 protein measurements for anthracycline benefit $[10,13]$ either as a stand-alone biomarker or in combination with $H E R 2$ or TOP $2 A$ gene aberrations. It should be mentioned, however, that in the original biomarker study of the BR9601, neither HER2 nor TOP2A gene aberrations were associated with benefit from anthracyclines [7].

Although only 288 of the original 374 patient samples were available for this study, survival analyses confirmed the benefit from the addition of an anthracycline to CMF versus CMF alone in this subset of patients. Moreover, when analyzing the associations between patient outcome and HER2 and TOP2A gene copy number and Ki-67 protein in the 288 patients, similar results were obtained as reported previously [7]. It thus appears that

Table 4 Unadjusted HR estimates of the treatment by marker effects

\begin{tabular}{|c|c|c|c|c|c|c|c|}
\hline & & RFS & & & os & & \\
\hline & & HR & $95 \% \mathrm{Cl}$ & TxM (HR) & HR & $95 \% \mathrm{Cl}$ & TxM (HR) \\
\hline \multirow[t]{2}{*}{ TIMP-1 } & Negative $(n=167 ; 58 \%)$ & 0.642 & 0.377 to 1.091 & 1.311 & 0.784 & 0.447 to 1.376 & 1.667 \\
\hline & Positive $(n=121 ; 42 \%)$ & 0.478 & 0.247 to 0.927 & (0.562 to 3.056$)$ & 0.461 & 0.213 to 1.002 & (0.641 to 4.339$)$ \\
\hline \multirow[t]{2}{*}{ 2T Profile } & Responsive ( $n=183 ; 66.8 \%)$ & 0.613 & 0.365 to 1.029 & 1.022 & 0.450 & 0.188 to 1.080 & 1.579 \\
\hline & Non-responsive $(n=91 ; 33.2 \%)$ & 0.582 & 0.277 to 1.221 & (0.415 to 2.520$)$ & 0.739 & 0.428 to 1.277 & (0.565 to 4.411$)$ \\
\hline \multirow[t]{2}{*}{ HT Profile } & Responsive ( $n=181 ; 66.1 \%)$ & 0.658 & 0.400 to 1.082 & 0.902 & 0.783 & 0.464 to 1.320 & 0.577 \\
\hline & Non-responsive $(n=93 ; 33.9 \%)$ & 0.531 & 0.235 to 1.200 & (0.347 to 2.346$)$ & 0.384 & 0.141 to 1.046 & (0.186 to 1.788$)$ \\
\hline
\end{tabular}




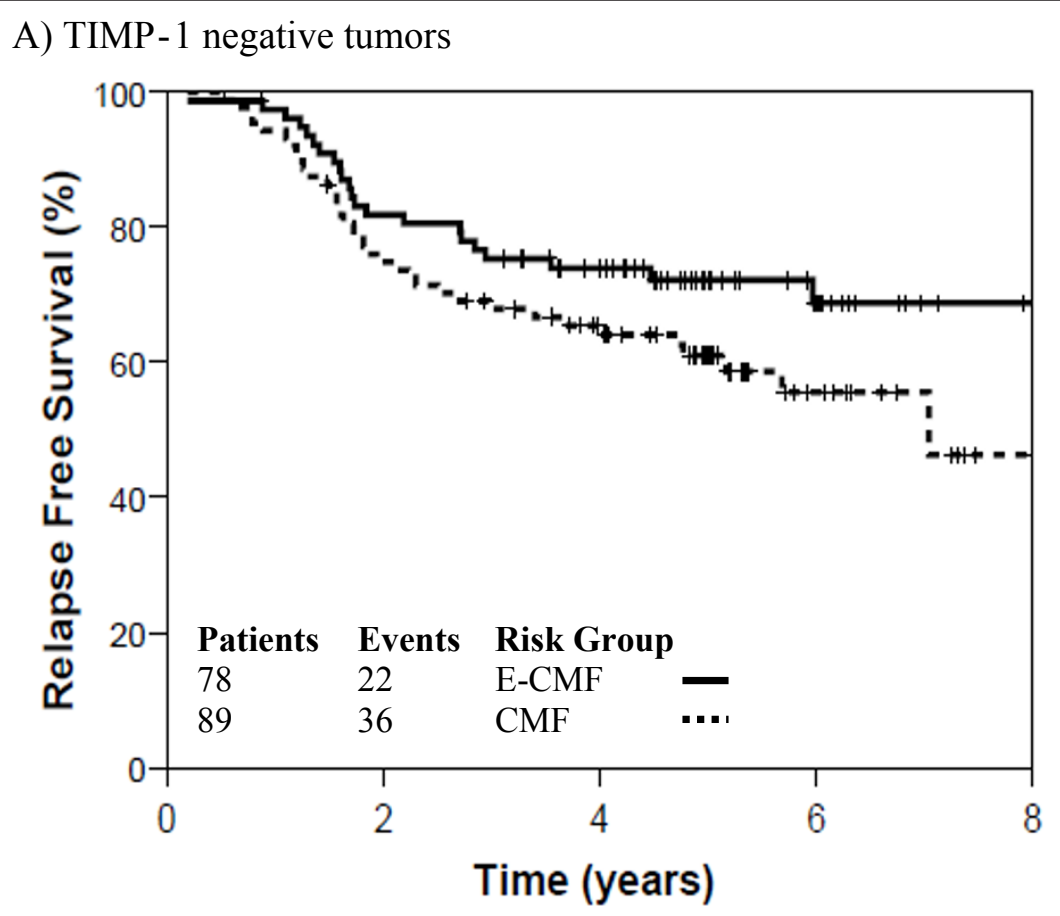

B) TIMP-1 immunoreactive tumors

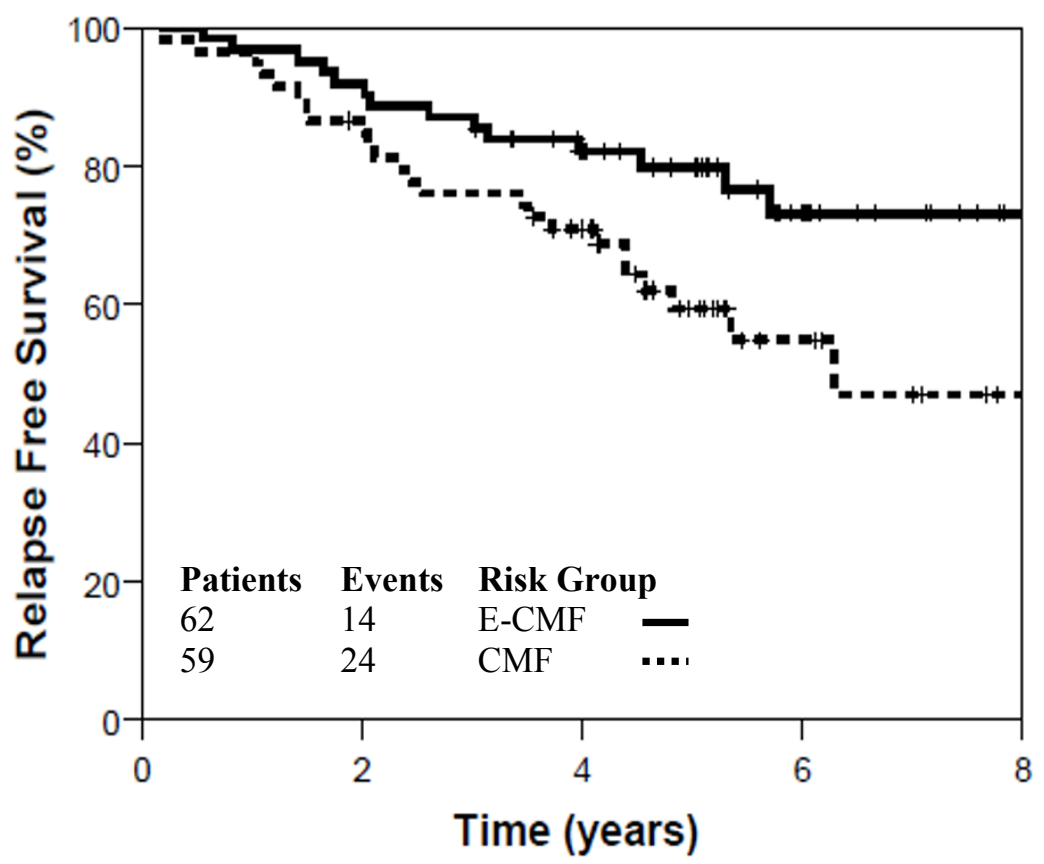

Figure 2 Relapse free survival for E-CMF (solid line) versus CMF (dashed line). Panel A: TIMP-1 negative tumors. Panel B: TIMP-1 immunoreactive tumors.

these 288 patients are representative for the initial study population.

The TIMP-1 immunostaining was performed using a validated anti-TIMP-1 monoclonal antibody and a strict protocol [18] and the scoring of TIMP-1 positivity/negativity was performed as previously described [10]. However, among the 288 patients $42 \%$ were found positive for TIMP-1, which is much less than what has previously 
been reported: in the DBCG 89D patient cohort 75\% of the cases were TIMP-1 positive [10] and a similar distribution between TIMP-1 positivity/negativity was seen in the MA5 study [14]. While TIMP-1 immunoreactivity is often heterogeneous [10] and smaller TMA might impact on results, the cores used here were similar to those used in the MA5 study. Also while in the DBCG 89D study [10], the cores were taken from the invasive front of the tumors while in the BR9601 and MA5 study sampling was focused on tumor rich areas. TIMP-1 immunoreactivity was significantly associated with lower Ki67 immunoreactivity, suggesting that TIMP-1 positive tumors have a lower rate of proliferation, which in turn might result in reduced sensitivity to chemotherapy. Conversely, in the present study in which TIMP-1 positive tumors had a non-significant increased benefit from the E-CMF combination as compared to CMF alone is not explained by increased proliferation of these tumors as evaluated by the Ki67 staining.

An alternative explanation for the discordant results between the present study and our previous studies $[10,13,14]$ is that the interaction depends on the regimens and/or the patient populations studied. The anthracycline regimens in the DBCG and the BR9601 studies are not identical, with a greater total dose and duration of epirubicin treatment in the DBCG study [3] as compared to BR9601 [2]. Furthermore, the DBCG trial recruited only pre-menopausal node-positive women, and patients did not receive adjuvant endocrine therapy. Conversely, in BR9601, both pre- and post-menopausal women were recruited, including $15 \%$ node negative tumors, and all ER positive cases were to receive five years of tamoxifen. Another noteworthy disparity between the trials was the higher percentage of ER positive patients recruited into BR9601 (54.9\%) compared to the DBCG trial (33.7\%). While these differences might explain the discordant results, there is no obvious a priori explanation as to why they should.

\section{Conclusions}

In conclusion, this validation study of TIMP-1 breast cancer cell immunoreactivity as a predictive biomarker for adjuvant anthracycline benefit did not support the use of this marker to select patients for anthracycline treatment. Moreover, this study could not confirm any additive predictive value by combining TIMP-1 immunoreactivity with results on HER2 and TOP2A FISH analyses.

\footnotetext{
Abbreviations

CEF: Cyclophosphamide, epirubicin, 5-fluorouracil; CEP17: Centromeric enumeration probe for chromosome 17; CMF: Cyclophosphamide, methotrexate, 5-fluorouracil; DFS: Disease free survival; E-CMF: EpirubicinCMF; ER: Estrogen receptor; FISH: Fluorescent in situ hybridization; HR: Hazard ratio; IHC: Immunohistochemistry; i.v.: Intravenous; OS: Overall survival; RFS: Relapse free survival; TIla: Topoisomerase Ila; TIMP-1: Tissue inhibitor of metalloproteinase 1; TMA: Tissue microarray.
}

Authors' contributions

JB, AM, DC, CT and NB designed the study. JB, DC, CT and AM collected the tissue samples, established the database and performed the statistical analysis. $E B$ and $A B$ performed and scored the TIMP-1 immunhistochemistry. AM, JB and NB interpreted the data and drafted the manuscript. All authors have read and approved the manuscript for publication.

\section{Competing interests}

The authors declare that they have no competing interests.

\section{Acknowledgements}

This study was supported by the Sino-Danish Breast Cancer Research Centre and A Race Against Breast Cancer. We are grateful to Jeremy Thomas for support in constructing the tissue micro-arrays.

This study complies with the current laws of the United Kingdom.

\section{Author details}

${ }^{1}$ Transformative Pathology, Ontario Institute for Cancer Research, MaRS Centre, South Tower, 101 College Street, Suite 800, Toronto, ON M5G 0A3, Canada. ${ }^{2}$ Section of Pathobiology, Institute of Veterinary Disease Biology, Faculty of Health and Medical Sciences, University of Copenhagen, Denmark. ${ }^{3}$ Department of Pathology, Herlev Hospital, Copenhagen, Denmark.

${ }^{4}$ University of Leeds and Cancer Research UK Centre, St James' University Hospital, Leeds, LS2 9JT, UK. ${ }^{5}$ Edinburgh Cancer Centre, Western General Hospital, Crewe Road South, Edinburgh EH4 2XU, UK. 'Biomarkers and Companion Diagnostics Group, Edinburgh Cancer Research UK Centre, MRC IGMM, University of Edinburgh, Crewe Road South, Edinburgh, EH4 2XR, UK.

Received: 21 July 2012 Revised: 19 December 2012

Accepted: 3 April 2013 Published: 9 April 2013

\section{References}

1. Levine MN, Pritchard KI, Bramwell VH, Shepherd LE, Tu D, Paul N, National Cancer Institute of Canada Clinical Trials Group: Randomized trial comparing cyclophosphamide, epirubicin, and fluorouracil with cyclophosphamide, methotrexate, and fluorouracil in premenopausal women with nodepositive breast cancer: update of National Cancer Institute of Canada Clinical Trials Group Trial MA5. J Clin Oncol 2005, 23:5166-5170.

2. Poole CJ, Earl HM, Hiller L, Dunn JA, Bathers S, Grieve RJ, Spooner DA, Agrawal RK, Fernando IN, Brunt AM, O'Reilly SM, Crawford SM, Rea DW, Simmonds P, Mansi JL, Stanley A, Harvey P, McAdam K, Foster L, Leonard RC, Twelves CJ: Epirubicin and cyclophosphamide, methotrexate, and fluorouracil as adjuvant therapy for early breast cancer. N Engl J Med 2006, 355:1851-1862.

3. Ejlertsen $B$, Mouridsen $H T$, Jensen MB, Andersen J, Cold S, Edlund $P$, Ewertz M, Jensen BB, Kamby C, Nordenskjold B, Bergh J: Improved outcome from substituting methotrexate with epirubicin: results from a randomised comparison of CMF versus CEF in patients with primary breast cancer. Eur J Cancer 2007, 43:877-884.

4. Bowles EA, Wellman R, Delate T, Allen L, Feigelson HS, Yood MU, Davis R, Nekhlyudov L, McCarty C, Habel L, Magid D, Onitilo A, Freedman A, Wagner E: C-B2-01: cardiotoxic chemotherapy is associated with increased heart failure risk among women with breast cancer in the cancer research network. Clin Med Res 2011, 9:148.

5. Gennari A, Sormani MP, Pronzato P, Puntoni M, Colozza M, Pfeffer U, Bruzzi P: HER2 status and efficacy of adjuvant anthracyclines in early breast cancer: a pooled analysis of randomized trials. J Natl Cancer Inst 2008, 100:14-20.

6. Knoop AS, Knudsen H, Balslev E, Rasmussen BB, Overgaard J, Nielsen KV Schonau A, Gunnarsdóttir K, Olsen KE, Mouridsen H, Ejlertsen B, Danish Breast Cancer Cooperative Group: Retrospective analysis of topoisomerase Ila amplifications and deletions as predictive markers in primary breast cancer patients randomly assigned to cyclophosphamide, methotrexate, and fluorouracil or cyclophosphamide, epirubicin, and fluorouracil: Danish Breast Cancer Cooperative Group. J Clin Oncol 2005, 23:7483-7490.

7. Bartlett JM, Munro A, Cameron DA, Thomas J, Prescott R, Twelves CJ: Type 1 receptor tyrosine kinase profiles identify patients with enhanced benefit from anthracyclines in the BR9601 adjuvant breast cancer chemotherapy trial. J Clin Oncol 2008, 26:5027-5035.

8. Leo AD, Desmedt C, Bartlett JM, Piette F, Ejlertsen B, Pritchard KI, Larsimont D, Poole C, Isola J, Earl H, Mouridsen H, O'Malley FP, Cardoso F, 
Tanner M, Munro A, Twelves CJ, Sotiriou C, Shepherd L, Cameron D, Piccart MJ, Buyse M: HER2 and TOP2A as predictive markers for anthracycline-containing chemotherapy regimens as adjuvant treatment of breast cancer: a meta-analysis of individual patient data. Lancet Oncol 2011, 12:1134-1142.

9. Pritchard KI, Munro A, O'Malley FP, Tu D, Li X, Levine MN, Shepherd L, Chia S, Bartlett JM: Chromosome 17 centromere (CEP17) duplication as a predictor of anthracycline response: evidence from the NCIC Clinical Trials Group (NCIC CTG) MA.5 Trial. Breast Cancer Res Treat 2011, 131:541-551.

10. Willemoe GL, Hertel PB, Bartels A, Jensen MB, Balslev E, Rasmussen BB, Mouridsen $\mathrm{H}$, Ejlertsen B, Brünner N: Lack of TIMP-1 tumour cell immunoreactivity predicts effect of adjuvant anthracycline-based chemotherapy in patients $(n=647)$ with primary breast cancer. A Danish Breast Cancer Cooperative Group Study. Eur J Cancer 2009, 45:2528-2536.

11. Davidsen ML, Wurtz SO, Romer MU, Sorensen NM, Johansen SK, Christensen IJ, Larsen JK, Offenberg H, Brunner N, Lademann U: TIMP-1 gene deficiency increases tumour cell sensitivity to chemotherapyinduced apoptosis. Br J Cancer 2006, 95:1114-1120.

12. Fu ZY, LV JH, Ma CY, Yang DP, Wang T: Tissue inhibitor of metalloproteinase-1 decreased chemosensitivity of MDA-435 breast cancer cells to chemotherapeutic drugs through the PI3K/AKT/NF-KB pathway. Biomed Pharmacother 2011, 65:163-167.

13. Ejlertsen $B$, Jensen MB, Nielsen KV, Balslev E, Rasmussen BB, Willemoe GL, Hertel PB, Knoop AS, Mouridsen HT, Brünner N: HER2, TOP2A, and TIMP-1 and responsiveness to adjuvant anthracycline-containing chemotherapy in high-risk breast cancer patients. J Clin Oncol 2010, 28:984-990.

14. Hertel PB, Tu D, Ejlertsen B, Jensen MB, Balslev E, Jiang S, O'Malley FP, Pritchard Kl, Shepherd LE, Bartels A, Brünner N, Nielsen TO: TIMP-1 in combination with HER2 and TOP2A for prediction of benefit from adjuvant anthracyclines in high-risk breast cancer patients. Breast Cancer Res Treat 2012, 132:225-234.

15. Bartlett JM, Going JJ, Mallon EA, Watters AD, Reeves JR, Stanton P, Richmond J, Donald B, Ferrier R, Cooke TG: Evaluating HER2 amplification and overexpression in breast cancer. J Pathol 2001, 195:422-428.

16. Ellis IO, Bartlett J, Dowsett M, Humphreys S, Jasani B, Miller K, Pinder SE, Rhodes A, Walker R: Best Practice No 176: updated recommendations for HER2 testing in the UK. J Clin Pathol 2004, 57:233-237.

17. Durbecq V, Paesmans M, Cardoso F, Desmedt C, Di Leo A, Chan S, Friedrichs K, Pinter T, Van Belle S, Murray E, Bodrogi I, Walpole E, Lesperance B, Korec S, Crown J, Simmonds P, Perren TJ, Leroy JY, Rouas G, Sotiriou C, Piccart M, Larsimont D: Topoisomerase-II alpha expression as a predictive marker in a population of advanced breast cancer patients randomly treated either with single-agent doxorubicin or single-agent docetaxel. Mol Cancer Ther 2004, 3:1207-1214.

18. Sorensen IV, Fenger C, Winther H, Foged NT, Lademann U, Brunner N, Usher PA: Characterization of anti-TIMP-1 monoclonal antibodies for immunohistochemical localization in formalin-fixed, paraffin-embedded tissue. J Histochem Cytochem 2006, 54:1075-1086.

19. Moller SN, Dowell BL, Stewart KD, Jensen V, Larsen L, Lademann U, Murphy G, Nielsen HJ, Brunner N, Davis GJ: Establishment and characterization of 7 new monoclonal antibodies to tissue inhibitor of metalloproteinases-1. Tumour Biol 2005, 26:71-80.

20. Dowsett M, Nielsen TO, A'Hern R, Bartlett J, Coombes RC, Cuzick J, Ellis M, Henry NL, Hugh JC, Lively T, MCShane L, Paik S, Penault-Llorca F, Prudkin L, Regan M, Salter J, Sotiriou C, Smith IE, Viale G, Zujewski JA, Hayes DF: Assessment of Ki67 in breast cancer: recommendations from the International Ki67 in Breast Cancer working group. J Natl Cancer Inst 2011, 103:1656-1664.

\section{doi:10.1186/bcr3411}

Cite this article as: Munro et al.: Is TIMP-1 immunoreactivity alone or in combination with other markers a predictor of benefit from anthracyclines in the BR9601 adjuvant breast cancer chemotherapy trial? Breast Cancer Research 2013 15:R31.

\section{Submit your next manuscript to BioMed Central and take full advantage of:}

- Convenient online submission

- Thorough peer review

- No space constraints or color figure charges

- Immediate publication on acceptance

- Inclusion in PubMed, CAS, Scopus and Google Scholar

- Research which is freely available for redistribution

Submit your manuscript at www.biomedcentral.com/submit
Biomed Central 